\title{
Study on Big Data Development of Test and Evaluation of Weapon and Equipment
}

\author{
Ke Hongfa *, Zhu Jilu, Du Hongmei \\ Department of Equipment Test, Equipment Academy, Beijing, China
}

\section{Email address:}

kehongfa2004@163.com (Ke Hongfa),sdzj12008@gmail.com (Zhu Jilu), donghuali000@sina.com (Du Hongmei)

${ }^{*}$ Corresponding author

\section{To cite this article:}

Ke Hongfa, Zhu Jilu, Du Hongmei. Study on Big Data Development of Test and Evaluation of Weapon and Equipment. Science Discovery. Vol. 4, No. 1, 2016, pp. 21-25. doi: 10.11648/j.sd.20160401.14

Received: February 18, 2016; Accepted: March 22, 2016; Published: April 8, 2016

\begin{abstract}
The big data development problem of test and evaluation of weapon and equipment is studied. Firstly, the concept of big data and research status in the military domain are summarized. And the concept of big data of test and evaluation of weapon and equipment is given. Secondly, the development demands and status of big data of test and evaluation of weapon and equipment are analyzed. Then, the development contents of big data of test and evaluation of weapon and equipment, such as data collection, big data calculation, data mining, and et al, are introduced. Finally, the key development problems of big data of test and evaluation of weapon and equipment, such as theory system, technique system, operation system, and et al, are proposed emphasisly.
\end{abstract}

Keywords: Equipment Test and Evaluation, Big Data, Development Contents, Key Development Problem

\section{武器装备试验鉴定的大数据建设问题研究}

\section{柯宏发", 祝冀鲁, 杜红梅}

装备试验系, 装备学院, 北京, 中国

\section{邮箱}

kehongfa2004@163.com（柯宏发）, sdzj12008@gmail.com（祝冀鲁）, donghuali000@sina. com（杜红梅）

摘要: 对武器装备试验鉴定的大数据建设问题进行了研究。概括了大数据的定义以及军事领域内大数据研究现状; 给 出了武器装备试验鉴定大数据的定义, 分析了装备试验鉴定大数据的建设需求和建设现状; 重点研究了装备试验鉴定 大数据的数据源采集、大数据计算、挖掘分析等建设内容, 以及理论体系、技术体系、运用体系等关键建设问题。

关键词：装备试验鉴定，大数据，建设内容，关键建设问题

\section{1. 引言}

随着新军事变革的不断推进, 世界各国都在不断探 索和研究武器装备战斗力快速形成是问题。武器装备试 验鉴定是武器装备全寿命管理中的一个重要阶段 $[1,2]$, 主要用来演示武器装备系统的可行性、评价设计风险、
寻求试验设计方案、评价装备作战需求的满意度等。武 器装备试验鉴定活动已经历经了几十年, 积累了大量的 试验数据资源, 这些数据既可作为武器装备作战效能、 作战适用性考核评估的依据, 也可作为装备论证方案分 析、仿真试验建模、试验方案设计、模型算法验证、装 备作战运用方式探索的驱动输入, 为武器装备的研制、 
设计、定型、鉴定、作战使用和技术支援保障提供数据 支撑。在大数据概念一路走红的时代背景下 [3-6], 研究 武器装备试验鉴定大数据建设问题、提高武器装备试验 鉴定数据运用质量是非常必要的, 并具有很强的现实意 义。目前, 武器装备试验鉴定数据的管理与控制还主要 是依靠各个试验组织单位, 面向通用性、基础性、共享 性的试验数据工程虽然处于建设之中, 但是数据资源建 设资源分散、数据权威性差、数据共享使用困难等矛盾 依然很突出, 与武器装备试验鉴定大数据建设要求还有 很远的差距。本文分析提出了武器装备试验鉴定大数据 的基本概念, 提出武器装备试验鉴定大数据建设的基本 内容及关键问题, 为武器装备试验鉴定大数据建设奠定 相关依据和提供支撑。

\section{2. 大数据的概念及研究现状}

\section{1. 大数据的概念}

大数据并不是一个新的概念, 该词最早出现在 20 世纪 90年代, 最近几年才引起各个行业的重视。美国政府 2012 年宣布《大数据研发倡议》 $[7]$, 旨在改进现有人们从海 量和复杂数据中获取知识的能力, 从而加速美国在科学与 工程领域创新的步伐, 由此掀起了全球大数据研究热潮。 麦肯锡全球研究所将 $[8]$ 大数据定义为 “一种规模大到在 获取、存储、管理、分析方面大大超出了传统数据库软件 工具能力范围的数据集集合”, 通常无法在可容忍的时间 内用传统的数据分析方法对其进行感知、获取、管理、处 理和服务 [9], 这个定义得到普遍的认同。大数据具有 $4 \mathrm{~V}$ 特征, 即数据体量 (Volume) 巨大、数据类型 (Variety) 繁多、流动速度 (Velocity) 快、价值 (Value) 密度低。 大数据分析的意义在于挖掘数据的价值。大数据问题成为 一个科学问题就是因为其 $4 \mathrm{~V}$ 特征, 大数据技术是指用以解 决大数据问题, 具体包括集成、存储、处理、分析、评估、 预测等方面在内的方法。大数据的分析具有两层涵义, 其 一是数据量巨大, 这是存储的问题, 其二是数据处理方法, 这是计算的问题。

\section{2. 军事领域大数据研究现状}

伴随着信息技术的高速发展, 世界主要军事强国很早 就开始关注数据建设问题, 纷纷将大数据纳入国家发展战 略, 以抢占大数据产业发展的制高点。为了维持在全球的 军事优势, 美国政府发布了《大数据研究和发展计划》, 美军也率先提出了数据战略的新概念, 不断加大对信息资 源建设和管理的投入，逐步通过计算中心、信息中心、服 务中心的建设解决数据建设和管理问题 $[10,11]$, 并取得 了较好的成效。目前美军已建立了依托网络环境的数据共 享体制, 实现了对装备全系统全寿命数据的信息化管理。 欧洲各国的武器装备数据资源建设基本上是以美军的系 统和技术为蓝本, 重点围绕作战数据的共享和使用问题, 结合本国的应用需求进行的。英国政府在大数据和节能计 算研究上投入巨资，积极推进数据开放计划。法国政府发 布了《数字化路线图》，宣布投入巨资研发大数据项目。
纵观军事强国的大数据建设现状和发展趋势, 试验 鉴定大数据建设是一项基础性、长期、艰巨的系统工程。 目前存在的突出问题是, 试验鉴定数据大多分散在各自 独立的数据库中, 没有形成系统完整的数据体系, 数据 的收集、积累、建设、使用等工作没有相关的管理机制 来保障常态化、正常化, 缺乏统筹规划、分步实施的试 验鉴定大数据建设途径来确保数据建设的可持续。缺少 配套的数据标准规范, 大量数据格式和定义不统一, 极 少的数据标准也没有得到贯彻执行, 数据建设和维护使 用无章可循; 缺乏统一的技术标准、技术手段和平台, 数据资源的集成很困难, 信息交互交换困难, 根本无法 实现交互共享。因此, 目前试验鉴定大数据建设的前提 条件不满足。

\section{3. 装备试验鉴定大数据的概念及建设需求}

\section{1. 试验鉴定大数据的定义}

武器装备试验鉴定大数据, 指的是武器装备单体、系 统、体系试验鉴定过程中所生成和积累的、无法用传统的 数据分析方法进行感知、获取、管理、处理和服务的数据 集合。试验鉴定大数据的数据量是一个不断变化的目标, 其规模、形式超出了传统数据处理方法所能捕获、管理和 处理的能力, 而且试验鉴定管理者目前处理数据能力的增 长速度远远跟不上数据量的增长速度。

试验鉴定大数据的 $4 V$ 特征比较明显, 一般包括以被试 装备组成、试验鉴定任务描述等为代表的结构化数据、以 试验大纲和试验报告等为代表的半结构化数据、以及以测 试数据、视频、语音信息为代表的非结构化等多类数据; 随着不同种类武器装备的增加, 通过不同的试验鉴定模式 产生了海量的试验鉴定数据; 试验鉴定数据资源的流动性 特别快; 试验鉴定大数据对于特定的装备来说, 其价值密 度比较低, 因为关于该装备性能、作战效能或作战适用性 的数据会淹没在大数据环境之中。

\section{2. 试验鉴定大数据建设需求}

试验鉴定大数据建设充满了机遇与挑战, 只有最快地 习惯大数据形式下的数据模式, 掌握和处理大数据处理方 法, 才能在装备论证、装备试验、装备保障、装备使用活 动中取得主动权。试验鉴定数据主要指武器装备在不同类 型的试验鉴定活动所需要用到的数据、生成的过程数据和 试验鉴定结果数据。

在装备论证活动中, 为了增强论证活动的有效性、可 信性、及时性等, 可以利用试验鉴定大数据中的相关历史 试验方法、条件、试验问题分析等数据, 结合被论证装备 所提的要求进行论证结果的检验和认定。

在装备试验活动中, 为了提高试验鉴定活动的效益, 可以利用试验鉴定大数据中的法规标准规程数据、试验手 段信息、装备的战技术指标和作战使用方式、作战使用环 境剖面数据相关的试验内容与方法等数据, 实物、半实物 仿真过程中产生的各类模型数据、试验评估数据等对装备 试验活动也同样具有较大的利用价值。 
装备保障工作主要包括装备的平时维护检测、战时应 急抢修与救援等, 因此, 试验鉴定大数据中有关装备的环 境适应性、可靠性、维修性、测试性、安全性、保障性等 六性数据、装备的故障信息数据以及装备的综合评价数据 等对装备保障工作也至关重要。

各种武器装备在试验鉴定活动中进行了大量的近实 战应用, 这些实战化考核隐含了装备在各种不同条件下的 作战能力, 典型作战任务剖面下的作战使用流程试验数据, 具体包括使用准备、作战实施、作战结束等各个阶段的性 能数据, 是武器装备实际作战使用中提高作战效能的重要 基础数据。

\section{3. 试验鉴定大数据建设现状}

由于试验鉴定大数据 $4 \mathrm{~V}$ 的存在, 肯定会对信息时代背 景下的数据处理产生很大的影响。过去那些装备试验鉴定 数据库、数据处理方式与方法开始有点不合时宜了, 需要 对试验鉴定数据处理进行理解方式和理念上的调整, 同时 也需要根据不同的试验鉴定评估需求, 采取不同的处理方 式和工具。因此, 抓好试验鉴定大数据建设及其实战化运 用, 对于提高武器装备作战效能和作战适用性评估、加强 装备体系的实战化运用具有重大的军事价值。

由于武器装备试验鉴定领域的特殊性, 面向服务的试 验鉴定数据的组织机构设置、运性维护管理流程不够科学, 缺乏统一完善的试验数据建设规章制度、技术规范和先进、 实用、高效的试验数据建设工具, 试验鉴定大数据建设严 重滞后于金融、商业等领域的大数据建设。

自上世纪信息化建设启动以来，装备管理相关部门 和单位在数据建设领域取得了很大成绩, 制定和颁布了 一系列数据建设标准规范, 但是离试验鉴定大数据建设 及其实战化运用要求还有较大的差距, 集中体现在以下 几个方面。一是试验鉴定大数据建设是一项知识密集、 技术复杂的系统工程, 目前总体管理水平太低, 缺乏顶
层总体规划, 缺少配套的日常数据建设与管理机制, 缺 少大数据建设可依据的成熟法规标准。二是试验鉴定大 数据建设基础太薄弱, 试验鉴定大数据来源分散, 来源 于科研论证、科研试验及地方工业部门等, 缺乏权威机 构的校核认证; 由于各个单位的基础条件建设和技术手 段等原因, 大量历史试验数据采用纸质、胶片等非数字 化方式进行存储; 而且由于各种武器装备型号及试验配 试设备型号众多, 导致试验数据资源种类和格式繁多, 在数据传递和使用过程中经常出现无标记、或者标记不 完整的现象。三是试验鉴定数据共享难度大, 受基础条 件建设、试验数据管理机制等多方面因素的制约, 试验 鉴定数据资源分散各个数据来源单位, 存储位置分散、 技术标准不统一, 难以形成完整、可靠的数据共享, 更 谈不上按需服务。四是数据的二次开发和应用缺失有效 手段, 试验鉴定数据深层次开发利用工作较少开展, 半 实物仿真试验数据缺乏与外场试验、数字仿真试验的数 据复用与融合处理, 试验组织单位同作战部队的交流脱 节造成试验数据利用率低, 对数据产品服务的指导作用 发挥不够, 多层次多视角的数据挖掘工作开展不够深入。

\section{4. 试验鉴定大数据的建设内容}

武器装备试验鉴定大数据的内容十分宽泛, 主要有 法规标准规程数据、试验鉴定组织机构和专家数据、被 试装备数据、试验鉴定数据、试验鉴定文件资料和试验 鉴定管理活动数据等。构建武器装备试验鉴定大数据平 台如图1所示, 对多种类、多来源、海量试验鉴定数据进 行自动、快速采集, 基于分布式计算特性和算法特性对 数据抽取、清洗等进行统一预处理 [9], 利用分布式计算 架构对分布式存储管理系统中的数据进行快速计算和挖 掘分析, 构建相应的装备作战效能评估、作战使用方式 分析等模型。

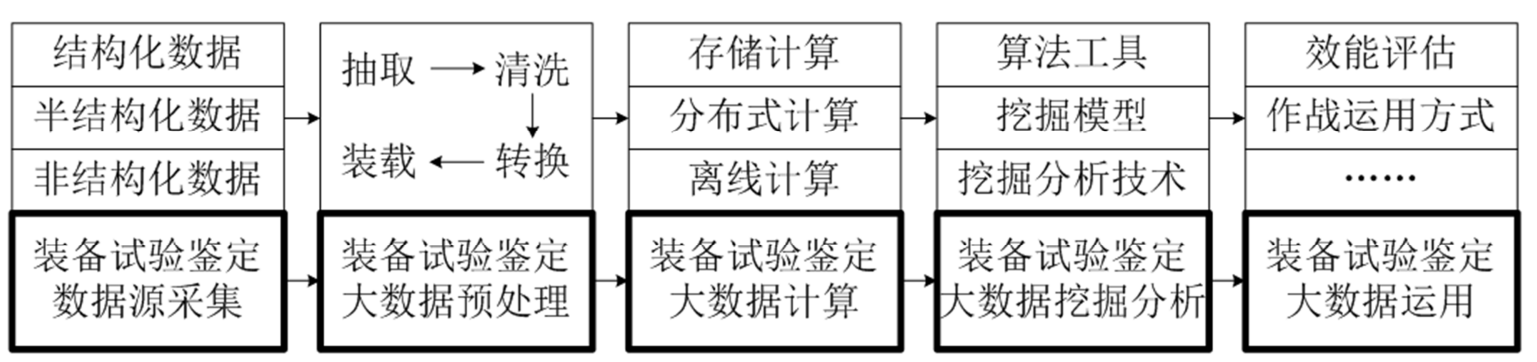

图1 研究内容结构图。
试验鉴定大数据根据数据类型的不同, 包括以装备系 统组成、试验鉴定任务描述等为代表的结构化数据, 以试 验大纲、实施方案和试验报告等为代表的半结构化数据, 以及以测试数据、视频、语音信息为代表的非结构化数据 等, 主要通过数据库读取、数据库导入等方式采集来源于 装备科研论证、装备科研试验及地方装备研制部门的数据 库信息以及相关的平面文件信息等。

试验鉴定大数据来源于不同的科研试验单位, 同一类 型的武器装备试验鉴定数据也可能来源于不同的体制编 制, 各种数据接口与描述方法千差万别, 导致原始数据显 得杂乱、重复、不完整等, 严重影响到后续数据计算和数
据挖掘算法的执行效率, 甚至最终会造成大数据分析结果 的南辕北辙。因此, 在采集试验鉴定原始数据之后, 首先 必须对其进行预处理。大数据预处理主要利用数据切片、 数据索引标记、数据聚合、数据分类等技术, 进行层级化 的提取、清洗、转换、聚合、重组等操作, 统一数据标准 和标准接口, 通过分布式存储管理技术建立多类型、多格 式、多特性数据的统一存储管理系统。

武器装备试验鉴定大数据建设需要建设一个分布式 计算平台, 该平台支持数据资源的动态管理与调度, 采用 分布式计算模型将数据分割成小块, 由信息共享的多台计 算机分别计算, 最后对运算结果进行统一合并。分布式计 
算框架可以采取分布式实时计算框架、分布式离线计算框 架或者两者相结合的框架模式。

通过上述几个步骤的分布式武器装备试验鉴定数据 管理系统, 其数据信息既可用于传统的查询、统计和分析, 也可以通过数据挖掘分析技术, 探索、分析和挖掘数据背 后的重要信息。大数据运用是建设武器装备试验鉴定大数 据的最终目的, 装备论证、装备试验、装备使用等阶段都 需要试验鉴定大数据的保障服务, 需要通过分布式计算平 台来充分利用蕴藏在数据中的信息和知识。

\section{5. 试验鉴定大数据建设的关键问题}

\section{1. 装备试验鉴定大数据理论体系}

武器装备试验鉴定理论是一门综合性的学科, 由装 备试验、系统工程、数据分析等分支学科组成, 装备试 验鉴定大数据更是一个全新的领域, 需要一个嵝新的理 论体系支撑其建设发展。目前的装备试验鉴定关注样本 采样, 总是从最少、精确的数据中能获取最多的信息,
基于因果关系寻求鉴定结果; 而装备试验鉴定数据除了 定量描述外, 更多的采用定性描述形式, 其形式多种多 样, 试验鉴定大数据需要关注全体数据, 从混杂的非结 构化数据中，更多的基于相关关系寻求鉴定结果。装备 试验鉴定大数据建设需要发展相关的大数据表示理论、 大数据计算理论、大数据挖掘理论及大数据安全运用理 论等, 其中每个理论的解决又需要很多相关理论的支撑。 例如, 装备试验鉴定大数据的挖掘过程如图2所示, 其挖 掘整个过程包含试验鉴定数据获取与存储、数据处理与 分析、数据挖掘、数据可视化及数据融合等, 该过程依 赖于基于大数据和知识库的智能推理等理论和技术支撑。 装备试验鉴定大数据挖掘具体过程所涉及理论包括试验 鉴定的数据的获取和存储理论, 大数据的去噪、采样、 过滤等处理理论, 试验鉴定数据的线性和非线性等统计 法分析理论, 数据间及数据类别间的关系理论, 试验鉴 定数据间的内在联系、隐含信息、模式及知识的人工神 经网络、决策树等理论与方法等等。

篮选

分类、集群

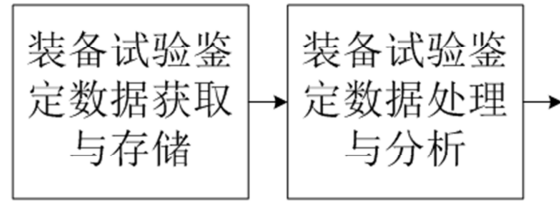

图2 装备试验鉴定大数据挖掘过程。

\section{2. 装备试验鉴定大数据技术体系}

面对装备试验鉴定的作战效能、作战适用性、体系贡 献率分析需求, 形成装备试验鉴定大数据工程的技术体系, 突出体现大数据技术体系的影响。由于大数据是非结构化 的, 需要发展新的大数据表示技术、面向要素的非结构化 数据库技术、数据更新技术和数据资源评价技术等。需要 基于云计算、增量计算等技术, 发展形成新的大数据计算 技术。需要引入数据挖掘、图匹配、网络分析等技术, 发 展形成新的大数据智能分析技术。需要引入数据的错误识 别、清洗、去午等技术, 形成新的大数据质量控制技术。 需要引入数据安全、动态监测、隐私保护等技术, 形成新 的大数据安全防护技术等。

\section{3. 装备试验鉴定大数据管理体系}

各种各样的武器装备全寿命管理阶段都在产生、利用 大数据, 因此迫切需要建立装备试验鉴定大数据管理体系, 通过建立相应的配套机制、制度, 实现试验鉴定大数据全 方位、全寿命的管理 $[12,13]$, 将各种数据工程纳入装备 试验鉴定大数据管理系统, 明确试验鉴定数据及其结构体 系、数据工程标准体系、数据工程的标准化接口等, 实现 装备训练运用、装备性能试验、装备作战试验数据资源的 整合, 使大数据建设成为装备论证、装备试验、装备使用 等相关单位的一项常态化工作。

\section{4. 装备试验鉴定大数据运用体系}

提供装备鉴定数据资源的实时、按需访问以及数据的 智能分析技术和数据挖掘技术是装备试验鉴定大数据建 设的根本目标，其实质在于最终的运用。装备试验鉴定大 数据不等于大信息、大信息也不等于大智慧, 必须对海量 的试验鉴定数据进行深度挖掘, 才能形成有利于装备全寿 命管理不同阶段所需要的有用信息。未来装备试验鉴定大 数据系统更多地应该采用面向智慧的服务模式, 通过装备 作战能力的数据抽取模型、装备作战能力的知识发现模型、 以及作战效能和作战适用性评估模型等, 务实推动装备试 验鉴定大数据的应用, 发挥装备试验鉴定大数据建设的整 体效益。

\section{6. 结束语}

在武器装备智能化、信息化、体系化发展的重要时期, 开展武器装备试验鉴定大数据问题研究是实现全面、科学 武器装备作战效能和作战适用性的重要手段, 也是解决装 备论证单位、装备使用单位无装备试验鉴定数据, 装备论 证单位、装备试验单位无装备作战使用数据等问题的有效 途径。论文给出了装备试验鉴定大数据的概念, 重点介绍 了其建设内容及关键问题, 下一步将需要对装备试验鉴定 
大数据建设的总体框架与系统组成、发展实施方案、相关 关键技术的解决方案等问题展开研究。

\section{致谢}

本文为部委级科研重点项目《电子装备作战试验设计 与规划》（ZS2014070130A12002）的阶段性成果之一。

\section{参考文献}

[1] 柯宏发, 黄彦昌, 罗剑. 对装备作战试验力量建设的思考 [J]. 装备学院学报, 2014, 25(2):60-64。

[2] 李高鹏. 装备试验数据资源应用现状及前景 $[J]$. 论证与研 究, $2014,30(4): 44-46$ 。

[3] 程莲娟. 美国推进大数据的应用实践及其有益借鉴 $[J]$. 情 报资料工作，2013 (5) : 110-112。

[4]《中国电子科学研究院学报》编辑部. 大数据时代 $[J]$. 中国 电子科学研究院学报, 2013, 8(1):27-31。

[5] 王珊, 王会举, 覃雄派, 等. 架构大数据: 挑战、现状与展望 $[J]$. 测绘学报, 2011，34(10):1741-1752。
[6] 曹凌. 大数据创新: 欧盟开放数据战略研究 $[J]$. 情报理论与 实践, $2013,36(4): 118-122$ 。

[7] The White House. Big data research and development initiative

[EB/OL]. http://www. whitehouse. gov/blog/2012/03/2 9/big-data-big-deal.

[8] McKinsey Global Institute. Big data: the next frontier for innovation, competition, and productivity [EB/OL]. http://www. mckinsey. com/insights/mgi/research/tech nology_and_innovation/big_data_the_next_frontier_f or_innovation.

[9] 孙雪萍, 崔久强. 大数据助力信息安全研究 $[J]$. 信息安全与 通信保密, 2015 (3) : 89-91。

[10] 李德仁, 张良培, 夏桂松. 遥感大数据自动分析与数据挖掘 $[J]$. 测绘学报, 2014, 43(12):1211-1216。

[11] 李清泉, 李德仁. 大数据GIS [J]. 武汉大学学报 ・信息科学版, 2014, 39 (6) :641-644。

[12] 丁建新, 樊江滨, 郭强, 等. 全周期试验数据管理系统研究 $[J]$. 车辆与动力技术, $2010(3): 4-10$ 。

[13] 董东, 朱成亮, 胡瑛, 等. 试验数据管理平台设计研究 $[\mathrm{J}]$. 火 箭推进, 2014, 40(4):67-72。 